Applied Mathematical Sciences, Vol. 7, 2013, no. 24, 1205 - 1208

HIKARI Ltd, www.m-hikari.com

\title{
Visual Landmarks Systems for Humanoid Robots
}

\author{
Anna Gorbenko \\ Department of Intelligent Systems and Robotics \\ Ural Federal University \\ 620083 Ekaterinburg, Russia \\ gorbenko.ann@gmail.com \\ Vladimir Popov \\ Department of Intelligent Systems and Robotics \\ Ural Federal University \\ 620083 Ekaterinburg, Russia \\ Vladimir.Popov@usu.ru
}

\begin{abstract}
In this paper, we consider a model of visual landmarks selection for humanoid robots navigation. This model is based on a genetic programming approach.
\end{abstract}

Keywords: humanoid robots, visual landmarks, mobile robot navigation

Problems of mobile robot navigation has remained as a subject of intense study for many decades (see e.g. [1] - [11]). In particular, visual landmarks systems has been extensively investigated for mobile robot navigation (see e.g. [12] - [16]). However, most of the well-known approaches to visual landmarks navigation were created and demonstrated on wheeled robots.

Note that many humanoid robots used visual landmarks systems. In particular, we can mention HRP-2 [17]. HRP-2 is fitted with a high-performance forward-looking trinocular camera rig. This camera provides the capability to make accurate 3D measurements in a focused observation area close in front of the robot. HRP-2 uses visual landmarks and monocular SLAM. A reinforcement learning method for visual landmarks navigating the Nao was presented in [18]. Reem-B uses two lasers [19]. Reem-B is able to construct a map and 
localize on it. Navigation of Reem-B is mainly guided by laser data. Visual landmarks used only as auxiliary method.

However, traditional approaches to visual landmarks navigation are insufficiently effective for humanoid robots. Usually, we need some additional constraints that keep some landmarks. In particular, the problem of placement of visual landmarks was proposed in [12]. The problem of selection of a minimal set of landmarks was proposed in [13]. This models are quite effective for wheeled robots. But, this models are insufficient reliable for humanoid robots. Humanoid robots have many more degrees of freedom. Even on a flat surface, a camera will have much variation in its positioning. For humanoid robots, we obtain much greater noise and difficulty in interpreting a stream of images.

For humanoid robots, we need redundant set of visual landmarks. This redundancy allows us to compensate the loss of some visual landmarks. We assume that

$$
\left(x_{i}, y_{i}, z_{i}\right)
$$

is a position of some landmark. In this case, we can consider a function

$$
\begin{gathered}
R_{i}\left(\left(x_{1}, y_{1}, z_{1}\right),\left(x_{2}, y_{2}, z_{2}\right), \ldots,\left(x_{m}, y_{m}, z_{m}\right)\right)= \\
\sum_{1 \leq j \leq t} \delta_{j} \prod_{i=1}^{m} x_{i}^{\alpha_{i, j}} \prod_{i=1}^{m} y_{i}^{\beta_{i, j}} \prod_{i=1}^{m} z_{i}^{\gamma_{i, j}}
\end{gathered}
$$

as a redundancy function for $\left(x_{i}, y_{i}, z_{i}\right)$.

We consider a redundancy function for $\left(x_{i}, y_{i}, z_{i}\right)$ as a unification of the probability of disappearance of landmark and the probability of usage of landmark. We consider

$$
t, \delta_{1}, \delta_{2}, \ldots, \delta_{t}, \alpha_{1,1}, \ldots, \alpha_{m, t}, \beta_{1,1}, \ldots, \beta_{m, t}, \gamma_{1,1}, \ldots, \gamma_{m, t}
$$

as chromosome and use a genetic algorithm to construct a set of redundancy functions. After this, we use a model of genetic programming based on the set of redundancy functions to construct a redundant set of visual landmarks. This model evolves a set of rules of the following form:

$$
\left[i ;\left\{R_{i} \mid i \in I\right\}\right] \rightarrow H_{i}=\left\{R_{i} \mid i \in J\right\}
$$

where $J \subseteq I$.

We assume that if a landmark $\left(x_{i}, y_{i}, z_{i}\right)$ needed for a humanoid robot localization, then the robot can use any landmark from $H_{i}$ instead $\left(x_{i}, y_{i}, z_{i}\right)$. Also, we assume that a robot can find at least one landmark from $H_{i}$ with a sufficiently high probability.

In our computational experiments, we have compared the quality of considered model of genetic programming based on the set of redundancy functions 


\begin{tabular}{|l|llll|}
\hline average number of generations & $10^{3}$ & $10^{4}$ & $10^{5}$ & $10^{6}$ \\
\hline number of landmarks for GP+GA & $46 \%$ & $32 \%$ & $2.1 \%$ & $0.7 \%$ \\
\hline number of landmarks for GA & $52 \%$ & $35 \%$ & $24 \%$ & $22 \%$ \\
\hline
\end{tabular}

Table 1: Average number of landmarks for GP+GA and GA.

\begin{tabular}{|l|llll|}
\hline average number of generations & $10^{3}$ & $10^{4}$ & $10^{5}$ & $10^{6}$ \\
\hline probability of failure for GP+GA & $7 \%$ & $6 \%$ & $0.32 \%$ & $0.07 \%$ \\
\hline probability of failure for GA & $3 \%$ & $2 \%$ & $1.9 \%$ & $1.63 \%$ \\
\hline
\end{tabular}

Table 2: Probability of failure for GP+GA and GA.

$(\mathrm{GP}+\mathrm{GA})$ and the quality of a genetic algorithm for direct prediction of a redundant set of visual landmarks (GA). Selected experimental results are given in Tables 1 and 2 .

ACKNOWLEDGEMENTS. The work was partially supported by Analytical Departmental Program "Developing the scientific potential of high school" 8.1616.2011.

\section{References}

[1] A. Gorbenko, M. Mornev, V. Popov, and A. Sheka, The problem of sensor placement for triangulation-based localisation, International Journal of Automation and Control, 5 (2011) 245-253.

[2] A. Gorbenko and V. Popov, On the Problem of Sensor Placement, Advanced Studies in Theoretical Physics, 6 (2012) 1117-1120.

[3] A. Gorbenko and V. Popov, Self-Learning Algorithm for Visual Recognition and Object Categorization for Autonomous Mobile Robots, Lecture Notes in Electrical Engineering, 107 (2012), 1289-1295.

[4] A. Gorbenko and V. Popov, Robot Self-Awareness: Usage of Co-training for Distance Functions for Sequences of Images, Advanced Studies in Theoretical Physics, 6 (2012) 1243-1246.

[5] A. Gorbenko and V. Popov, Robot's Actions and Automatic Generation of Distance Functions for Sequences of Images, Advanced Studies in Theoretical Physics, 6 (2012) 1247-1251.

[6] A. Gorbenko and V. Popov, Anticipation in Simple Robot Navigation and Finding Regularities, Applied Mathematical Sciences, 6 (2012) 6577-6581. 
[7] A. Gorbenko and V. Popov, Footstep Planning for Humanoid Robots, Applied Mathematical Sciences, 6 (2012) 6567-6571.

[8] A. Gorbenko and V. Popov, Multi-agent Path Planning, Applied Mathematical Sciences, 6 (2012) 6733-6737.

[9] A. Gorbenko and V. Popov, Programming for Modular Reconfigurable Robots, Programming and Computer Software, 38 (2012) 13-23.

[10] A. Gorbenko, M. Mornev, and V. Popov, Planning a Typical Working Day for Indoor Service Robots, IAENG International Journal of Computer Science, 38 (2011) 176-182.

[11] A. Gorbenko, V. Popov, and A. Sheka, Localization on Discrete Grid Graphs, Lecture Notes in Electrical Engineering, 107 (2012), 971-978.

[12] A. Gorbenko and V. Popov, On the Problem of Placement of Visual Landmarks, Applied Mathematical Sciences, 6 (2012), 689-696.

[13] A. Gorbenko and V. Popov, The Problem of Selection of a Minimal Set of Visual Landmarks, Applied Mathematical Sciences, 6 (2012), 4729-4732.

[14] A. Gorbenko and V. Popov, A Real-World Experiments Setup for Investigations of the Problem of Visual Landmarks Selection for Mobile Robots, Applied Mathematical Sciences, 6 (2012), 4767-4771.

[15] A. Gorbenko and V. Popov, Computational Experiments for the Problem of Selection of a Minimal Set of Visual Landmarks, Applied Mathematical Sciences, 6 (2012), 5775-5780.

[16] V. Popov, Partially Distinguishable Guards, Applied Mathematical Sciences, 6 (2012) 6587-6591.

[17] O. Stasse, Andrew Davison, R. Sellaouti, and K. Yokoi, Real-time 3D SLAM for Humanoid Robot considering Pattern Generator Information IEEE/RSJ International Conference on Intelligent Robots and Systems, (2006), 348-355.

[18] S. Oßwald, A. Hornung, and M. Bennewitz, Learning reliable and efficient navigation with a humanoid, IEEE International Conference on Robotics and Automation, (2010), 2375-2380.

[19] R. Tellez, F. Ferro, S. Garcia, E. Gomez, E. Jorge, D. Mora, D. Pinyol, J. Oliver, O. Torres, J. Velazquez, and D. Faconti, 8th IEEE-RAS International Conference on Humanoid Robots, (2008), 462-468.

\section{Received: December 3, 2012}

